\title{
Water-insoluble fractions of botanical foods lower blood ethanol levels in rats by physically maintaining the ethanol solution after ethanol administration
}

\section{Shunji Oshima, Sachie Shiiya, Tomomasa Kanda}

Research and Development Laboratories for Innovation, Asahi Group Holdings, Ltd., Ibaraki, Japan

Corresponding author: Shunji Oshima, $\mathrm{PhD}$, Research and Development Laboratories for Innovation, Asahi Group Holdings, Ltd., 1-21, Midori 1-chome, Moriya-shi, Ibaraki, 302-0106, Japan

Submission Date: July 8, 2015, Acceptance date: November 29, 2015: Publication date: November 30, 2015

\begin{abstract}
Background: Several studies have analyzed the functions of foods and dietary constituents in the dynamics of alcohol metabolism. However, few studies have reported the function of dietary fibers in the dynamics of alcohol metabolism.
\end{abstract}

Objective: We assessed the effects of botanical foods that contain dietary fibers on alcohol metabolism.

Methods: The ability of the water-insoluble fraction (WIF) of 18 kinds of botanical foods to maintain $15 \%(\mathrm{v} / \mathrm{v})$ ethanol solution was examined using easily handled filtration. A simple linear regression analysis was performed to examine the correlation between the filtered volumes and blood ethanol concentration (BEC) in F344 rats $4 \mathrm{~h}$ after the ingestion of $4.0 \mathrm{~g} / \mathrm{kg}$ of ethanol following dosage of $2.5 \%(\mathrm{w} / \mathrm{v})$ WIF of the experimental botanical foods. Furthermore, the supernatant (6.3 Brix; water-soluble fraction) and precipitate (WIF of tomato), with a strong ethanol-maintaining ability, were obtained and BEC and the residual gastric ethanol in rats were determined $2 \mathrm{~h}$ after the administration of $4.0 \mathrm{~g} / \mathrm{kg}$ of ethanol and the individuals fractions. 
Results: The filtered volumes of dropped ethanol solutions containing all the botanical foods tested except green peas were decreased compared with the ethanol solution without WIF (control). There was a significant correlation between the filtered volumes and blood ethanol concentration (BEC). There was no significant difference in the residual gastric ethanol between controls and the supernatant group; however, it was increased significantly in the WIF group than in controls or the supernatant group. Consistent with this, BEC reached a similar level in controls and the supernatant group but significantly decreased in the WIF group compared with controls or the supernatant group.

Conclusions: These findings suggest that WIFs of botanical foods, which are mostly water-insoluble dietary fibers, possess the ability to absorb ethanol-containing solutions, and this ability correlates strongly with the inhibition of the blood ethanol response.

Key words: Botanical food, tomato, water-insoluble fraction, dietary fiber, blood ethanol concentration

\section{BACKGROUND}

Several studies have analyzed the functions of foods and dietary constituents in the dynamics of alcohol metabolism following their oral ingestion. Alcohol and its metabolites exert various physiological effects in the human body [1-3]; therefore, elucidating the functions of foods or dietary components in alcohol pharmacokinetics is very important to regulate alcohol levels and thereby reduce the harmful effects of alcohol or its metabolites on human health. Many dietary compounds have been employed to increase the rate of alcohol metabolism in vivo, including amino acids, carbohydrates, and vitamins. For example, oral fructose or sucrose stimulates the elimination of alcohol from the blood stream of healthy subjects or alcoholics [4-6]. Pyruvate increases the oxidation of alcohol, an effect that was attributed a coupled oxidation-reduction reaction between alcohol and pyruvate. Moreover, the conversion of alanine to pyruvate by oxidative deamination is a well-known metabolic reaction, and it was reported that the ability of alanine to decrease the blood ethanol concentration (BEC) could be attributed to the formation of pyruvate [7]. The ingestion of corn peptide rich in alanine reduced the increase in BEC after alcohol intake by markedly increasing plasma alanine and leucine levels compared with the water ingestion control group [8].

The metabolic pathways described above generate NAD+, which facilitates alcohol 
oxidation in the liver via the conversion of pyruvate to lactate. However, the most important effect of food on the dynamics of alcohol is the prolonged retention of alcohol in the stomach. It is now well established that the rate of gastric emptying is a major determinant of alcohol absorption. After the ingestion of meals, gastric emptying is paced to deliver nutrients to the duodenum [9]. Thus, alcohol consumed after food high in calories, regardless of the nutritional composition (e.g., high fat, high protein, or high carbohydrate), is likely released into the duodenum more slowly, thereby reducing the rate of absorption and attenuating the subsequent BEC [10]. In addition, alcohol may be metabolized before it reaches the systemic circulation because it can be removed presystemically via "first pass metabolism" [11]. The first pass metabolism of alcohol is likely to be modulated significantly by the gastric emptying rate. A previous study suggested that delayed gastric emptying increases the time that alcohol is exposed to gastric $\mathrm{ADH}$, and thereby increases the gastric first pass metabolism of alcohol. In addition, the hepatic first pass metabolism of alcohol may be enhanced as a result of the slower absorption of alcohol from the small intestine [12].

Most social alcohol drinking occurs with concurrent food consumption; therefore, we consider that the effect of dietary components on the gastric emptying rate is very important to regulate the dynamics of alcohol metabolism. Dietary fibers can reduce the glycemic index at least in part by the delaying gastric emptying [13]. However, to the best of our knowledge, few studies have assessed the effects of dietary fibers on the dynamics of alcohol metabolism.

Thus, in this study, we assessed the effects of botanical foods that contain dietary fibers on alcohol metabolism. Data revealed a strong lowering effect of water-insoluble fractions (WIFs) (predominantly water-insoluble dietary fibers) of botanical foods on BEC after ethanol administration in almost direct proportion to physically maintaining ethanol solution.

\section{MATERIALS AND METHODS}

\section{Materials}

Ethanol was obtained from Kanto Chemical Co., Inc. (Tokyo, Japan). Commercially available purees of vegetables, fruits, and legumes, including apples, asparagus, broccoli, burdock, corn, green peas, mangoes, peaches, pears, pimento (green, red, and yellow), pumpkin, strawberries, and tomato paste, were kindly provided by Kagome Co., Ltd. (Tokyo, Japan). Cabbage paste and carrot puree were purchased from Danfoods Co., Ltd. (Aichi, Japan). Concentrated orange juice was provided by Asahi Soft Drinks Co., Ltd. (Tokyo, Japan).

\section{Preparation of water-insoluble fractions}


One kilogram of each botanical food puree or paste was added to $3 \mathrm{~L}$ of water, stirred thoroughly, and then centrifuged at 3,000 rpm for $10 \mathrm{~min}$. The supernatant was then removed carefully to eliminate water-soluble constituents. The washes were repeated until the Brix value in the supernatant solution reached 0 . The precipitates were then frozen and freeze-dried to obtain a WIF of each vegetable and fruit in the form of dry powder. The resulting powders were stored in a desiccator attached to a vacuum aspirator at room temperature until their ethanol-maintaining ability was assessed or until use in Experiment 1.

\section{Preparation of water-soluble or insoluble fractions of tomato paste}

For Experiment 2, $35 \mathrm{~g}$ of tomato paste was diluted using distilled water to a final volume of 100 $\mathrm{mL}$, stirred thoroughly, and centrifuged at 3,000 rpm for $20 \mathrm{~min}$. The resulting supernatant and precipitate were diluted using distilled water to $100 \mathrm{~mL}$ and washed again; the precipitate was washed three times. This yielded $100 \mathrm{~mL}$ of supernatant solution (6.3 Brix) as the water-soluble fraction of diluted tomato paste $(9.0$ Brix) and $100 \mathrm{~mL}$ of precipitate as WIF. The precipitate contained $1.0 \%(\mathrm{w} / \mathrm{v}) \mathrm{WIF}$ fraction. Samples were stored at $5^{\circ} \mathrm{C}$ until use in Experiment 2.

\section{Ethanol-maintaining ability of water-insoluble fractions}

It is known that polysaccharides exhibit water-absorbing properties [14]. However, it is unclear whether they can also absorb alcohol or alcohol-containing solutions. Therefore, easily handled filtration experiments were performed to examine the ethanol-maintaining ability of WIFs of some vegetables and fruits. Briefly, solutions containing $0.5 \%(\mathrm{w} / \mathrm{v}) \mathrm{WIF}$ of each botanical food and $15 \%(\mathrm{v} / \mathrm{v})$ ethanol in distilled water were prepared. A circular filter paper (110 mm diameter, Tokyo Roshi Kaisha Ltd., Tokyo, Japan) was placed on a cone-shaped funnel, and $10 \mathrm{~mL}$ of the each fluid specimen (or $15 \%$ ethanol solution as the control) were poured on the filter paper at room temperature under atmospheric conditions. After $5 \mathrm{~min}$, the filtered volume $(\mathrm{mL})$ of ethanol solution was analyzed three times.

\section{Animal studies}

Animal studies were performed in accordance with the ethical guidelines for animal care, and the animal care committee of Asahi Group Holdings Ltd. (Ibaraki, Japan) approved the experimental protocol. F344 male rats were obtained from Japan SLC Inc. (Hamamatsu, Japan). The animals were housed in a room maintained at $25^{\circ} \mathrm{C}$ with $55 \%$ relative humidity, and were given ad libitum access to laboratory chow pellets and tap water in individual stainless steel cages until 
use for the experiments.

For Experiment 1, 4-6 rats weighing 180-250 g per group were fasted overnight and assigned to groups that received one of the 18 WIFs of the different botanical foods or to the control group. All rats were given $20 \mathrm{~mL} / \mathrm{kg}$ of either water containing $2.5 \%(\mathrm{w} / \mathrm{v}) \mathrm{WIF}$ or water alone (control) orally. They were then given $30 \%$ ethanol solution twice (total ethanol dose, 4.0 $\mathrm{g} / \mathrm{kg}$ ): once $30 \mathrm{~min}$ later and once $60 \mathrm{~min}$ later. Four hours after the oral administration of alcohol, blood samples were collected from the tail vein of each rat. This time point was selected because we had established that BEC peaked approximately $4 \mathrm{~h}$ after the administration of alcohol via this method by our preliminary experiments [15].

In Experiment 2, male F344 rats ( $\mathrm{n}=4$ /group) weighing 260-360 g were fasted for $24 \mathrm{~h}$ and then assigned randomly to groups that received $20 \mathrm{~mL} / \mathrm{kg}$ of either the water-soluble fraction or WIF of diluted tomato paste or water (control) orally. They were then given $30 \%$ ethanol solution twice (total ethanol dose, $4.0 \mathrm{~g} / \mathrm{kg}$ ): once $30 \mathrm{~min}$ later and once $60 \mathrm{~min}$ later. Blood samples were then collected from the large vein of each rat after $2 \mathrm{~h}$, and the stomach was ligated immediately at both the cardiac and pyloric regions and harvested under carbon dioxide anesthesia. The total fluids in the stomach were diluted using distilled water to a total volume of $50 \mathrm{~mL}$. The residual ethanol in the stomach was calculated as a percentage of the total ethanol dose.

\section{Measuring BEC and residual ethanol in the stomach}

The amount of ethanol in the blood and residual fluid in the stomach was measured using headspace gas chromatography as described previously [16]. Briefly, blood or stomach fluid samples $(50 \mu \mathrm{L})$ were treated immediately with $2.5 \mathrm{~mL}$ of ice-cold $4 \%(\mathrm{w} / \mathrm{v})$ perchloric acid (PCA) with ethanol-d6 added as an internal standard in a capped tube. After centrifugation at $3,000 \mathrm{rpm}$ for $10 \mathrm{~min}$ at $4^{\circ} \mathrm{C}, 2.0 \mathrm{~mL}$ of supernatant was transferred to a glass vial containing $0.50 \mathrm{~g}$ of solid $\mathrm{NaCl}$, and the PCA-treated samples were applied to a GC-MS system. The GC-MS analysis was performed using an Agilent $6890 \mathrm{~N}$ gas chromatography instrument coupled with an Agilent MS-5975C inert XL mass selective detector and an Agilent headspace sampler G1888 (Agilent Technologies, Little Fall, NY, USA). A capillary DB-WAX column (60 $\mathrm{m} \times 0.25 \mathrm{~mm}$, Agilent J \& W GC Columns) was used to separate the ethanol peak fraction. The initial temperature of $35^{\circ} \mathrm{C}$ was maintained for $3 \mathrm{~min}$, was increased to $80^{\circ} \mathrm{C}$ at a rate of $4^{\circ} \mathrm{C} / \mathrm{min}$, was increased to $240^{\circ} \mathrm{C}$ at a rate of $20^{\circ} \mathrm{C} / \mathrm{min}$, and then maintained at $240^{\circ} \mathrm{C}$ for $4 \mathrm{~min}$. The split ratio was 1:20, and helium was used as a carrier gas at a flow rate of $1.0 \mathrm{~mL} / \mathrm{min}$. The injector 
and detector temperatures were $210^{\circ} \mathrm{C}$ and $250^{\circ} \mathrm{C}$, respectively. The ethanol and ethanol-d6 (Merck, 99\% D anhydrous) peaks in the treated samples were identified by comparisons with the retention times and mass spectra of known standards.

\section{Statistical analyses}

Simple linear regression (Pearson's) analysis was performed to examine the correlation between BEC $(\mathrm{mg} / \mathrm{mL})$ and the filtered volume $(\mathrm{mL})$ of each WIF of experimental botanical foods to assess the relationship between blood alcohol lowering effects and the ethanol-maintaining ability. The relationship was calculated as a correlation coefficient (r) using Dr. SPSS II software (SPSS Inc.). Differences in BEC or residual alcohol in the stomach among groups in the animal study were analyzed using one-way analysis of variance (ANOVA) followed by Tukey's HSD test. Means with the same superscript letter are not significantly different $(p<0.05)$.

\section{RESULTS}

The ability of WIF of each of the 18 botanical foods to maintain a $15 \%(\mathrm{v} / \mathrm{v})$ ethanol solution is shown in Figure 1. During filtration, the ethanol solution in the fluids dripped through the filter paper more slowly when WIF exhibited strong ethanol-maintaining ability. The filtered volume of dropped ethanol solution over $5 \mathrm{~min}$ as the control was $5.47 \pm 0.12 \mathrm{~mL}$. The volumes of the ethanol solutions containing all botanical foods except for green peas were decreased compared with those of the control. Moreover, the volumes varied greatly among the botanical foods. In particular, we observed that a smaller filtered volume of the WIF of mangoes or tomatoes had a stronger ethanol-maintaining ability $(0.23 \pm 0.06 \mathrm{~mL}$ and $0.30 \pm 0.01 \mathrm{~mL}$, respectively).

We next performed simple linear regression (Pearson's) analysis to examine the correlation between the filtered volumes and the BEC of rats that received the WIF of experimental botanical foods $4 \mathrm{~h}$ after ethanol ingestion. As shown in Figure 2, the correlation coefficient between the filtered volume and BEC was $r=0.862$, and the two parameters were significantly correlated $(p<0.001)$.

The residual ethanol in the stomach and BEC $2 \mathrm{~h}$ after the administration of ethanol in Experiment 2 are presented in Table 1. The residual gastric ethanol in water controls, the water-soluble fraction group, and the WIF group was $26.8 \%, 23.7 \%$, and $51.9 \%$, respectively. There was no significant difference between controls and the water-soluble fraction group; however, the amount of gastric ethanol was increased significantly in the WIF group compared with controls and the supernatant group. The BEC in controls and the supernatant group was 
$3.30 \mathrm{mg} / \mathrm{mL}$ and $3.14 \mathrm{mg} / \mathrm{mL}$, respectively, $2 \mathrm{~h}$ after the administration of $4.0 \mathrm{~g} / \mathrm{kg}$ of ethanol.

However, BEC was $1.88 \mathrm{mg} / \mathrm{mL}$ in the WIF group, which was decreased significantly compared with that in controls and the supernatant group.

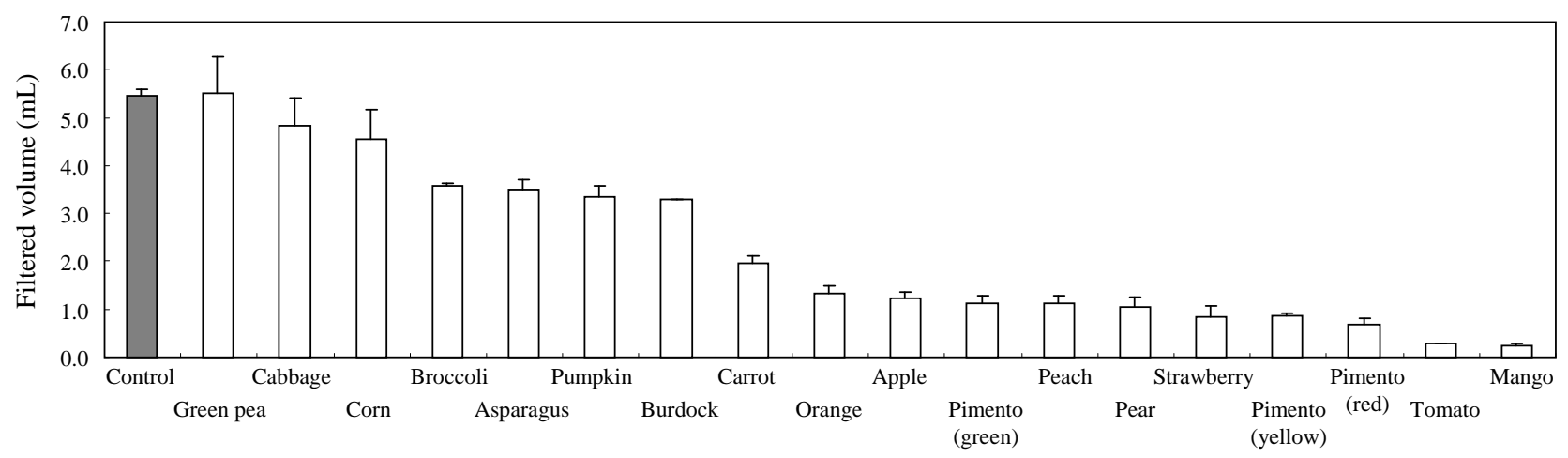

Figure 1. The ability of water-insoluble fractions of each of the 18 botanical foods to maintain $15 \%$ (v/v) ethanol solution. Data are expressed means and standard deviations. The volumes of the ethanol solutions containing all botanical foods except for green peas were decreased compared with those of the control. Moreover, the volumes varied greatly among the botanical foods.

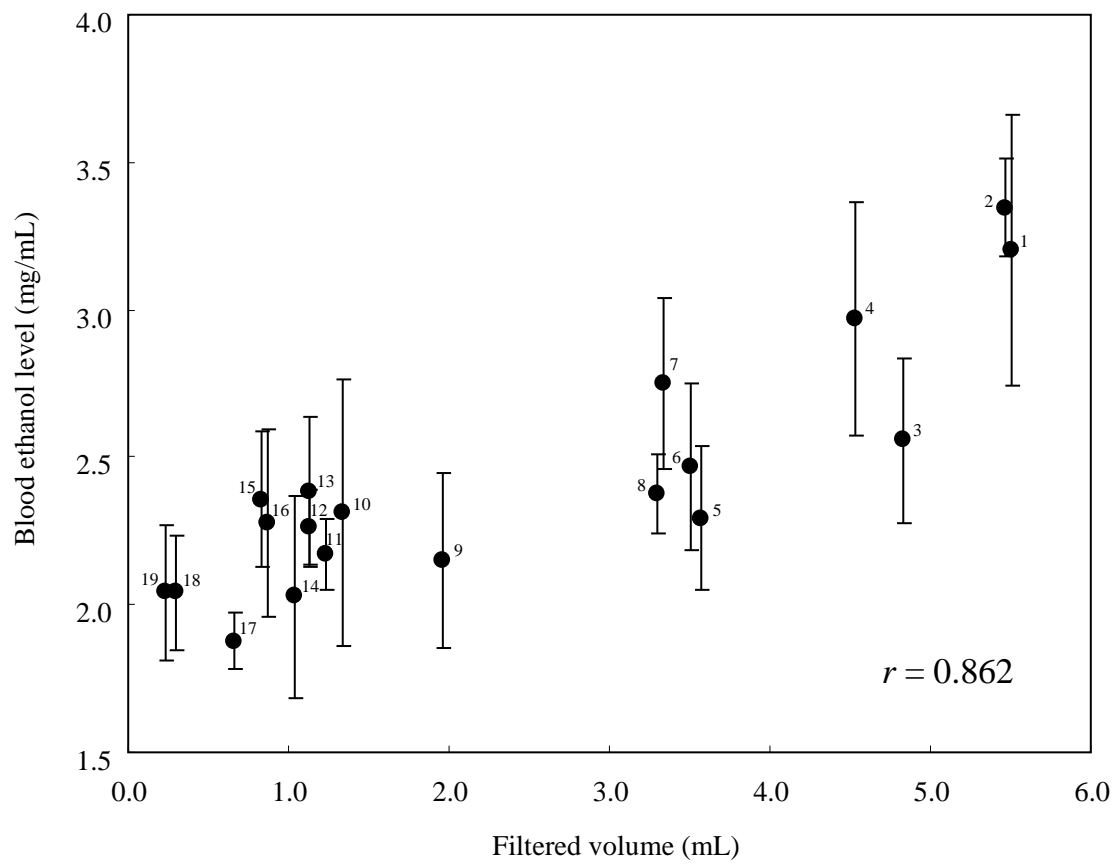

Figure 2. The correlation between the filtered ethanol volumes and blood ethanol concentration. The numbers indicated following the water-insoluble fractions of each of the botanical foods: 1.control, 2.green pea, 3.cabbage, 4.corn, 5.broccoli, 6.asparagus, 7.pumpkin, 8.burdock, 9.carrot, 10.orange, 11.apple, 12.pimento (green), 13.peach, 14.pear, 15.strawbwrry, 16.pimento (yellow), 17.pimento (red), 18.tomato, and 19.mango. Blood ethanol levels (mg/mL) are expressed as 
means and standard deviations, and the filtered volumes $(\mathrm{mL})$ are expressed as means. It was performed simple linear regression (Pearson's) analysis to examine the correlation between the filtered volumes and the BEC of rats that received the WIF of experimental botanical foods $4 \mathrm{~h}$ after ethanol ingestion. The correlation coefficient between the filtered volume and BEC was $r=$ 0.862 , and the two parameters were significantly correlated $(p<0.001)$.

Table 1. Residual ethanol in the stomach and the blood ethanol concentration $2 \mathrm{~h}$ after the administration of $4.0 \mathrm{~g} / \mathrm{kg}$ of ethanol

\begin{tabular}{lrr}
\hline & Residual amount of ethanol in stomach & BEC \\
\hline Control & $26.8(6.4)^{\mathrm{a}}$ & $3.30(0.55)^{\mathrm{a}}$ \\
$\begin{array}{l}\text { Diluted tomato paste (adjusted Brix 9.0) supernatant } \\
\text { (as water-soluble fraction) } \\
\text { Precipitation (as water-insoluble fraction) }\end{array}$ & $23.7(4.9)^{\mathrm{a}}$ & $3.14(0.66)^{\mathrm{a}}$ \\
\hline
\end{tabular}

Results are expressed as means and standard deviation (mean \pm SD). Means with different superscript lowercase letters are significantly different $(p<0.05)$ among groups.

\section{DISCUSSION}

To the best of our knowledge, no previous studies have focused on the ethanol-absorbing properties of dietary fibers, though it is well known water-absorbing actions of those components [17]. In addition, the blood alcohol-lowering effects of dietary fibers in vivo likely involve delaying gastric emptying. The current study revealed that WIFs of botanical foods, which are mostly water-insoluble dietary fibers, possessed the ability to absorb ethanol-containing solutions. This ability strongly correlated with the inhibition of the blood ethanol response, probably by delaying gastric emptying. It is known that the ingestion of food with alcohol delays the absorption of the alcohol, and that this is essentially due to the effects of food on gastric emptying. The current study confirmed that precipitates containing the WIF of tomatoes delayed the gastric emptying rate of ethanol solutions after oral administration to rats. Crude WIFs with water-soluble constituents that were removed were examined in the current study because of the difficulties associated with purifying insoluble dietary fibers from many kinds of botanical foods. However, no reports that indicated small amounts of insoluble micronutrients, minerals, or lipids in WIF possess the ability to absorb ethanol-containing solutions. Therefore, the effects of WIF were likely due to insoluble dietary fibers.

Previous studies showed that the content of dietary fibers, which consist of cellulose, lignin, 
hemicellulose, pectins, gums, and mucilages, differed between raw and cooked botanical foods [18-22]. In addition, the cellulose and hemicellulose contents of vegetables were greatly reduced because of prolonged heating [23]. Therefore, WIFs of the botanical foods used in the current study were prepared by freeze-drying because the ethanol-absorbing actions may lower due to heating. Nevertheless, the results suggest that raw or mildly heated botanical foods rich in dietary fibers should be investigated further as potential dietary components for lowering blood alcohol levels in humans.

In the current study, the ethanol-absorbing properties and BEC lowering effects of WIFs of tomatoes were stronger than those of WIFs of other botanical foods, except for mangoes. However, Ushida et al. reported that the concentrated aqueous fraction of tomato decreased BEC in rats [24]. The difference between these results may be caused by the degree of the condensation (Brix) of aqueous components in each tomato sample. This suggests that when alcohol is consumed after tomato intake, the WIF in tomatoes effectively decreases BEC, at least compared with consuming alcohol under fasting conditions, even if an inadequate amount of the aqueous components of tomatoes have been consumed. Specifically, the present study, the oral administration of $4.0 \mathrm{~g} / \mathrm{kg}$ of ethanol in the fasted state resulted in a BEC of $3.30 \mathrm{mg} / \mathrm{mL}$. However, the same dose of ethanol following the administration of WIF of the diluted tomato paste resulted in a $\mathrm{BEC}$ of $1.88 \mathrm{mg} / \mathrm{mL}$. Additional high quality studies are warranted to determine the effective amount of WIF of botanical foods in humans who consume alcohol to extrapolate the dosage of WIF from rat to human to reduce or ameliorate a drunkenness condition following the consumption of alcohol. The novel findings of the present study may be useful to help regulate the dynamics of alcohol metabolism using the dietary fibers in commonly consumed foods and to reduce some of the negative effects of alcohol or its metabolites on human health.

\section{CONCLUSIONS}

These results suggest that WIFs of botanical foods, which are mostly water-insoluble dietary fibers, possess the ability to absorb ethanol-containing solutions. In addition, this ability correlates strongly with the inhibition of the blood ethanol response. This is a previously unknown function of water-insoluble dietary fibers in common foods.

List of Abbreviations used: blood ethanol concentration (BEC), water-insoluble fraction (WIF) 
Competing Interest: The authors have the following interests. Shunji Oshima, Sachie Shiiya and Tomomasa Kanda are employed by Research \& Development Laboratories for Innovation, Asahi Group Holdings, Ltd., Ibaraki, Japan. There are no products in development or marketed products to declare.

Author's Contributions: All authors contributed to the study.

Acknowledgements: The authors wish to thank Akihiro Nemoto, Chiaki Hirota, Ema Toki and Hiroko Ishikiriyama for technical support in this study. In addition, the authors would like to thank Enago (www.enago.jp) for the English language review.

\section{REFERENCES}

1. Standridge JB, Zylstra RG, Adams SM.: Alcohol consumption: an overview of benefits and risks. South Med J 2004, 97: 664-672.

2. Foster RK, Marriott HE.: Alcohol consumption in the new millennium - weighing up the risks and benefits for our health. Nutr Bull 2006, 31: 286-331.

3. Guo R Ren J.: Alcohol and acetaldehyde in public health: from marvel to menace. Int $J$ Environ Res Public Health 2010, 7: 1285-1301.

4. Soterakis J, Iber FL.: Increased rate of alcohol removal from blood with oral fructose and sucrose. Am J Clin Nutr 1975, 28: 254-257.

5. Rawat AK.: Effects of fructose and other substances on ethanol and acetaldehyde metabolism in man. Res Commun Mol Pathol Pharmacol 1977, 16: 281-290.

6. Uzuegbu UE, Onyesom I.: Fructose-induced increase in ethanol metabolism and the risk of Syndrome X in man. Comptes Rendus Biologies 2009, 332: 534-538.

7. Westerfeld WW, Stotz E, Berg RL.: The role of pyruvate in the metabolism of ethyl alcohol. J Biol Chem 1942, 144: 657-665.

8. Yamaguchi M, Nishikiori F, Ito M. Furukawa Y.: The effects of corn peptide ingestion on facilitating alcohol metabolism in healthy men. Biosci Biotech Biochem 1997, 61: 1474-1481.

9. Hunt JN, Stubbs DF.: The volume and energy content of meals as determinants of gastric emptying. J Physiol 1975, 245: 209-225.

10. Jones AW, Jönsson KA, Kechagias S.: Effect of high-fat, high-protein, and high-carbohydrate meals on the pharmacokinetics of a small dose of ethanol. Br J Clin 
Pharmacol 1997, 44: 521-526.

11. Lieber CS, Gentry RT, Baraona E.: First pass metabolism of ethanol. Alcohol Alcohol 1994, (Suppl. 2): 163-169.

12. Oneta CM, Simanowski UA, Martinez M, Allali-Hassani A, Parés X, Homann N, Conradt C, Waldherr R, Fiehn W, Coutelle C, Seitz HK.: First pass metabolism of ethanol is strikingly influenced by the speed of gastric emptying. Gut 1998, 43: 612-619.

13. Scazzina F, Siebenhandl-Ehn S, Pellegrini N.: The effect of dietary fibre on reducing the glycaemic index of bread. Br J Nutr 2013, 109: 1163-1174.

14. Eastwoood MA.: Vegetable fibre: its physical properties. Proc Nutr Soc 1973, 32: 137-143.

15. Oshima S, Shiiya S, Tokumaru T, Kanda T.: Alanine with the precipitate of tomato juice administered to rats enhances the reduction in blood ethanol levels. J Nutr Metab 2015, article ID 280781.

16. Okada T, Mizoi Y.: Studies on the problem of blood acetaldehyde determination in man and level after alcohol intake. Jpn J Alcohol \& Drug Dependence 1982, 17: 141-159.

17. Boulos NN, Greenfield H, Wills RBH.: Water holding capacity of selected soluble and insoluble dietary fibre. Int J Food Prop 2000, 3: 217-231.

18. Herranz JA, Vidal-Valverde C, Rojas-Hidalgo E.: Cellulose, hemicellulose and lignin content of raw and cooked processed vegetables. J Food Sci 1981, 46: 1927-1933.

19. Mongeau R, Brassard R.: Determination of neutral detergent fiber in breakfast cereals: pentose, hemicellulose, cellulose and lignin content. J Food Sci 1982, 47: 550-555.

20. Lund ED, Smoot JM.: Dietary fiber content of some tropical fruits and vegetables. $J$ Agric Food Chem 1982, 30: 1123-1127.

21. Holloway WD.: Composition of fruit, vegetable and cereal dietary fibre. J Sci Food Agric 1983, 34: 1236-1240.

22. Ross JK, English C, Perlmutter CA.: Dietary fiber constituents of selected fruits and vegetables. J Am Diet Assoc 1985, 85: 1111-1116.

23. Rehman ZU, Islam M, Shah WH.: Effect of microwave and conventional cooking on insoluble dietary fibre components of vegetables. Food Chem 2003, 80: 237-240.

24. Ushida Y, Oshima S, Aizawa K, Suganuma H, Nemoto A, Ishikiriyama H, Kitagawa Y.: Aqueous components of tomato accelerate alcohol metabolism by increasing pyruvate level. FNS 2014, 5: 870-879. 